\title{
A Modular Equational Generalization Algorithm $^{\star}$
}

\author{
María Alpuente ${ }^{1}$, Santiago Escobar ${ }^{1}$, José $_{\text {Meseguer }}^{2}$, and Pedro Ojeda ${ }^{1}$ \\ 1 Universidad Politécnica de Valencia, Spain. \\ $\{$ alpuente, sescobar, pojeda\}@dsic.upv.es \\ 2 University of Illinois at Urbana-Champaign, USA. meseguer@cs.uiuc.edu
}

\begin{abstract}
This paper presents a modular equational generalization algorithm, where function symbols can have any combination of associativity, commutativity, and identity axioms (including the empty set). This is suitable for dealing with functions that obey algebraic laws, and are typically mechanized by means of equational atributes in rule-based languages such as ASF+SDF, Elan, OBJ, Cafe-OBJ, and Maude. The algorithm computes a complete set of least general generalizations modulo the given equational axioms, and is specified by a set of inference rules that we prove correct. This work provides a missing connection between least general generalization and computing modulo equational theories, and opens up new applications of generalization to rule-based languages, theorem provers and program manipulation tools such as partial evaluators, test case generators, and machine learning techniques, where function symbols obey algebraic axioms. A Web tool which implements the algorithm has been developed which is publicly available.
\end{abstract}

\section{Introduction}

The problem of ensuring termination of program manipulation techniques arises in many areas of computer science, including automatic program analysis, synthesis, verification, specialisation, and transformation. An important component for ensuring termination of these techniques is a generalization algorithm (also called anti-unification) that, for a pair of input expressions, returns its least general generalization (lgg), i.e., a generalization that is more specific than any other such generalization. Whereas unification produces most general unifiers that, when applied to two expressions, make them equivalent to the most general common instance of the inputs [21], generalization abstracts the inputs by computing their most specific generalization. As in unification, where the most general unifier (mgu) is of interest, in the sequel we are interested in the least general generalization (lgg) or, as we shall see for the equational case treated in this paper, in a minimal and complete set of lggs, which is the dual analogue of a minimal and complete set of unifiers for equational unification problems [6].

\footnotetext{
* This work has been partially supported by the EU (FEDER) and the Spanish MEC TIN2007-68093-C02-02 project, UPV PAID-06-07 project, and Generalitat Valenciana GV06/285 and BFPI/2007/076 grants; and by NSF Grant CNS 07-16638.
} 
For instance, in the partial evaluation (PE) of logic programs [17], the general idea is to construct a set of finite (possibly partial) deduction trees for a set of initial calls, and then extract from those trees a new program $P$ that allows any instance of the calls to be executed. To ensure that the partially evaluated program covers all these calls, most PE procedures recursively specialize some calls that are dynamically produced during this process. This requires some kind of generalization in order to enforce the termination of the process: if a call occurring in $P$ that is not sufficiently covered by the program embeds an already evaluated call, both calls are generalized by computing their lgg. In the literature on partial evaluation, least general generalization is also known as most specific generalization (msg) and least common anti-instance (lcai) [24].

The computation of lggs is also central to most program synthesis and learning algorithms such as those developed in the area of inductive logic programming [25], and also to conjecture lemmas in inductive theorem provers such as Nqthm [10] and its ACL2 extension [20]. Least general generalization was originally introduced by Plotkin in [28], see also [31]. Actually, Plotkin's work originated from the consideration in [30] that, since unification is useful in automatic deduction by the resolution method, its dual might prove helpful for induction. Anti-unification is also used in test case generation techniques to achieve appropriate coverage [7].

Quite often, however, all the above applications of generalization may have to be carried out in contexts in which the function symbols satisfy certain equational axioms. For example, in rule-based languages such as ASF+SDF [8], Elan [9], OBJ [18], CafeOBJ [14], and Maude [11] some function symbols may be declared to obey given algebraic laws (the so-called equational attributes of OBJ, CafeOBJ and Maude), whose effect is to compute with equivalence classes modulo such axioms while avoiding the risk of non-termination. Similarly, theorem provers, both general first-order logic ones and inductive theorem provers, routinely support commonly occurring equational theories for some function symbols such as associativity-commutativity. In yet another area, $[15,16]$ describes rule-based applications to security protocol verification, where symbolic reachability analyses modulo algebraic properties allow one to reason about security in the face of attempted attacks on low-level algebraic properties of the functions used in the protocol (e.g. commutative encryption). A survey of algebraic properties used in cryptographic protocols can be found in [13].

Surprisingly, unlike the dual case of equational unification, which has been thoroughly investigated (see, e.g., the surveys $[32,6]$ ), to the best of our knowledge there seems to be no treatment of generalization modulo an equational theory $E$. This paper makes a novel contribution in this area by developing a modular family of E-generalization algorithms where the theory $E$ is parametric: any binary function symbol in the given signature can have any combination of associativity, commutativity, and identity axioms (including the empty set of such axioms).

To better motivate our work, let us first recall the standard generalization problem. Let $t_{1}$ and $t_{2}$ be two terms. We want to find a term $s$ that generalizes 
both $t_{1}$ and $t_{2}$. In other words, both $t_{1}$ and $t_{2}$ must be substitution instances of $s$. Such a term is, in general, not unique. For example, let $t_{1}$ be the term $f(f(a, a), b)$ and let $t_{2}$ be $f(f(b, b), a)$. Then $s=x$ trivially generalizes the two terms, with $x$ being a variable. Another possible generalization is $f(x, y)$, with $y$ being also a variable. The term $f(f(x, x), y)$ has the advantage of being the most 'specific' or least general generalization (lgg) (modulo variable renaming).

In the case where the function symbols do not satisfy any algebraic axioms, the $\operatorname{lgg}$ is unique up to variable renaming. However, when we want to reason modulo certain axioms for the different function symbols in our problem, lggs no longer need to be unique. This is analogous to equational unification problems, where in general there is no single mgu, but a set of them. Let us, for example, consider that the above function symbol $f$ is associative and commutative. Then the term $f(f(x, x), y)$ is not the only least general generalization of $f(f(a, a), b)$ and $f(f(b, b), a)$, because another incomparable generalization exists, namely, $f(f(x, a), b)$.

Similarly to the case of equational unification [32], things are not so easy as for syntatic generalization, and the dual problem of computing least general $E$-generalizations is a difficult one, particularly in managing the algorithmic complexity of the problem. The significance of equational generalization was already pointed out by Pfenning in [27]: "It appears that the intuitiveness of generalizations can be significantly improved if anti-unification takes into account additional equations which come from the object theory under consideration. It is conceivable that there is an interesting theory of equational anti-unification to be discovered". In this work, we do not address the $E$-generalization problem in its fullest generality. Instead, we study in detail a modular algorithm for a parametric family of commonly occurring equational theories, namely, for all theories $(\Sigma, E)$ such that each binary function symbol $f \in \Sigma$ can have any combination of the following axioms: (i) associativity $\left(A_{f}\right) f(x, f(y, z))=f(f(x, y), z)$; (ii) commutativity $\left(C_{f}\right) f(x . y)=f(y, x)$, and (iii) identity $\left(U_{f}\right)$ for a constant symbol, say, $e$, i.e., $f(x, e)=x$ and $f(e, x)=x$. In particular, $f$ may not satisfy any such axioms, which when it happens for all binary symbols $f \in \Sigma$ gives us the standard generalization algorithm as a special case.

\section{Our contribution}

The main contributions of the paper can be summarized as follows:

- A modular equational generalization algorithm specified as a set of inference rules, where different function symbols satisfying different associativity and/or commutativity and/or identity axioms have different inference rules. To the best of our knowledge, this is the first equational least general generalization algorithm in the literature.

- Correctness and termination results for our $E$-generalization algorithm.

- A prototypical implementation of the $E$-generalization algorithm which is publicly available. 
The algorithm is modular in the precise sense that the combination of different equational axioms for different function symbols is automatic and seamless: the inference rules can be applied to generalization problems involving each symbol with no need whatsoever for any changes or adaptations. This is similar to, but much simpler and easier than, modular methods for combining $E$-unification algorithms (e.g., [6]). We illustrate our inference system with several examples.

As already mentioned, our $E$-generalization algorithm should be of interest to developers of rule-based languages, theorem provers and equational reasoning programs, as well as program manipulation tools such as program analyzers, partial evaluators, test case generators, and machine learning tools, for declarative languages and reasoning systems supporting commonly occurring equational axioms such as associativity, commutativity and identity efficiently in a built-in way. For instance, this includes many theorem provers, and a variety of rulebased languages such as ASF+SDF, OBJ, CafeOBJ, Elan, and Maude.

\section{Related work}

Although generalization goes back to work of Plotkin [28], Reynolds [31], and Huet [19], and has been studied in detail by other authors (see for example the survey [21]), to the best of our knowledge, we are not aware of any existing equational generalization algorithm modulo any combination of associativity, commutativity and identity axioms. While Plotkin [28] and Reynolds [31] gave imperative-style algorithms for generalization, which are both essentially the same, Huet's generalization algorithm was formulated as a pair of recursive equations [19]. Least general generalization in an order-sorted typed setting was studied in [1]. In [3], we specified the generalization process by means of an inference system and then extended it naturally to order-sorted generalization. Pfenning [27] gave an algorithm for generalization in the higher-order setting of the calculus of constructions which does not consider either order-sorted theories or equational axioms.

\section{Plan of the Paper}

After some preliminaries in Section 2, we present in Section 3 a syntactic generalization algorithm as a special case to motivate its equational extension. Then in Section 4 we show how this calculus naturally extends to a new, modular generalization algorithm modulo ACU. We illustrate the use of the inference rules with several examples. Finally, we prove the correctness of our inference system. Section 5 concludes. Proofs of the technical results can be found in [2].

\section{Preliminaries}

We follow the classical notation and terminology from [33] for term rewriting and from $[22,23]$ for rewriting logic. We assume an unsorted signature $\Sigma$ with a finite number of function symbols. We assume an enumerable set of variables $\mathcal{X}$. 
A fresh variable is a variable that appears nowhere else. We write $\mathcal{T}(\Sigma, \mathcal{X})$ and $\mathcal{T}(\Sigma)$ for the corresponding term algebras. For a term $t$, we write $\operatorname{Var}(t)$ for the set of all variables in $t$. The set of positions of a term $t$ is written $\operatorname{Pos}(t)$, and the set of non-variable positions $\operatorname{Pos}_{\Sigma}(t)$. The root position of a term is $\Lambda$. The subterm of $t$ at position $p$ is $\left.t\right|_{p}$ and $t[u]_{p}$ is the term $t$ where $\left.t\right|_{p}$ is replaced by $u$. By $\operatorname{root}(t)$ we denote the symbol occurring at the root position of $t$.

A substitution $\sigma$ is a mapping from a finite subset of $\mathcal{X}$, written $\operatorname{Dom}(\sigma)$, to $\mathcal{T}(\Sigma, \mathcal{X})$. The set of variables introduced by $\sigma$ is $\operatorname{Ran}(\sigma)$. The identity substitution is $i d$. Substitutions are homomorphically extended to $\mathcal{T}(\Sigma, \mathcal{X})$. The application of a substitution $\sigma$ to a term $t$ is denoted by $t \sigma$. The restriction of $\sigma$ to a set of variables $V$ is $\left.\sigma\right|_{V}$. Composition of two substitutions is denoted by juxtaposition, i.e., $\sigma \sigma^{\prime}$. We call a substitution $\sigma$ a renaming if there is another substitution $\sigma^{-1}$ such that $\left.\sigma \sigma^{-1}\right|_{D o m(\sigma)}=i d$.

A $\Sigma$-equation is an unoriented pair $t=t^{\prime}$. An equational theory $(\Sigma, E)$ is a set of $\Sigma$-equations. An equational theory $(\Sigma, E)$ is regular if for each $t=t^{\prime} \in E$, we have $\operatorname{Var}(t)=\operatorname{Var}\left(t^{\prime}\right)$. Given $\Sigma$ and a set $E$ of $\Sigma$-equations, equational logic induces a congruence relation $=_{E}$ on terms $t, t^{\prime} \in \mathcal{T}(\Sigma, \mathcal{X})$ (see [23]).

The $E$-subsumption preorder $\leq_{E}$ (simply $\leq$ when $E$ is empty) holds between $t, t^{\prime} \in \mathcal{T}(\Sigma, \mathcal{X})$, denoted $t \leq_{E} t^{\prime}$ (meaning that $t$ is more general than $t^{\prime}$ modulo $E$ ), if there is a substitution $\sigma$ such that $t \sigma={ }_{E} t^{\prime}$; such a substitution $\sigma$ is said to be an $E$-matcher for $t^{\prime}$ in $t$. The $E$-renaming equivalence $t \simeq_{E} t^{\prime}$ (or $\simeq$ if $E$ is empty), holds if there is a renaming $\theta$ such that $t \theta={ }_{E} t^{\prime}$. We write $t<_{E} t^{\prime}$ (or $<$ if $E$ is empty) if $t \leq_{E} t^{\prime}$ and $t \varkappa_{E} t^{\prime}$.

\section{Syntactic Least General Generalization}

In this section we revisit syntactic generalization [19], giving a novel inference system that will be useful in our subsequent extension of this algorithm to the equational setting given in Section 4.

Most general unification of a (unifiable) set $M$ is the least upper bound (most general instance) of $M$ under $\leq$. Generalization corresponds to the greatest lower bound. Given a non-empty set $M$ of terms, the term $w$ is a generalization of $M$ if, for all $s \in M, w \leq s$. A term $w$ is the least general generalization (lgg) of $M$ if $w$ is a generalization of $M$ and, for each other generalization $u$ of $M, u \leq w$.

The non-deterministic generalization algorithm $\lambda$ of Huet [19] (also treated in detail in [21]) is as follows. Let $\Phi$ be any bijection between $\mathcal{T}(\Sigma, \mathcal{X}) \times \mathcal{T}(\Sigma, \mathcal{X})$ and a set of variables $V$. The recursive function $\lambda$ on $\mathcal{T}(\Sigma, \mathcal{X}) \times \mathcal{T}(\Sigma, \mathcal{X})$ that computes the lgg of two terms is given by:

$-\lambda\left(f\left(s_{1}, \ldots, s_{m}\right), f\left(t_{1}, \ldots, t_{m}\right)\right)=f\left(\lambda\left(s_{1}, t_{1}\right), \ldots, \lambda\left(s_{m}, t_{m}\right)\right)$, for $f \in \Sigma$

$-\lambda(s, t)=\Phi(s, t)$, otherwise.

Central to this algorithm is the global function $\Phi$ that is used to guarantee that the same disagreements are replaced by the same variable in both terms.

In [3], we have provided a novel set of inference rules for computing the (syntactic) least generalization of two terms, that uses a local store of already 
solved generalization sub-problems. The advantage of using such a store is that, differently from the global repository $\Phi$, our stores are local to the computation traces. This non-globality of the stores is the key for both, the order-sorted version of [3] and the equational generalization algorithm developed in this work, which computes a complete and minimal set of least general $E$-generalizations.

In our reformulation [3], we represent a generalization problem between terms $s$ and $t$ as a constraint $s \stackrel{x}{\triangleq}$, where $x$ is a fresh variable that stands for the (most general) generalization of $s$ and $t$. By means of this representation, any generalization $w$ of $s$ and $t$ is given by a suitable substitution $\theta$ such that $x \theta=w$.

We compute the least general generalization of $s$ and $t$, written $\lg g(s, t)$, by means of a transition system $($ Conf,$\rightarrow)$ [29] where Conf is a set of configurations and the transition relation $\rightarrow$ is given by a set of inference rules. Besides the constraint component, i.e., a set of constraints of the form $t_{i} \triangleq x_{i} t_{i^{\prime}}$, and the substitution component, i.e., the partial substitution computed so far, configurations also include an extra component, called the store.

Definition 1. A configuration $\langle C T|S| \theta\rangle$ consists of three components: (i) the constraint component $\mathrm{CT}$, i.e., a conjuntion $s_{1} \triangleq x_{1} \triangleq t_{1} \wedge \ldots \wedge s_{n} \triangleq x_{n} \triangleq t_{n}$ that represents the set of unsolved constraints, (ii) the store component $\mathrm{S}$, that records the set of already solved constraints, and (iii) the substitution component $\theta$, that consists of bindings for some variables previously met during the computation.

Starting from the initial configuration $\left\langle t \triangleq t^{\prime} t^{\prime}|\emptyset| i d\right\rangle$, configurations are transformed until a terminal configuration $\langle\emptyset|S| \theta\rangle$ is reached. Then, the lgg of $t$ and $t^{\prime}$ is given by $x \theta$. As we shall see, $\theta$ is unique up to renaming.

The transition relation $\rightarrow$ is given by the smallest relation satisfying the rules in Figure 1. In this paper, variables of terms $t$ and $s$ in a generalization problem $t \triangleq s$ are considered as constants, and are never instantiated. The meaning of the rules is as follows.

- The rule Decompose is the syntactic decomposition generating new constraints to be solved.

- The rule Recover checks if a constraint $t \triangleq x_{\triangleq} s \in C T$ with $\operatorname{root}(t) \not \equiv \operatorname{root}(s)$, is already solved, i.e., if there is already a constraint $t \triangleq s \in S$ for the same conflict pair $(t, s)$, with variable $y$. This is needed when the input terms of the generalization problem contain the same conflict pair more than once, e.g., the $\operatorname{lgg}$ of $f(a, a, a)$ and $f(b, b, a)$ is $f(y, y, a)$.

- The rule Solve checks that a constraint $t \triangleq s \in C T$ with $\operatorname{root}(t) \not \equiv \operatorname{root}(s)$, is not already solved. If not already there, the solved constraint $t \triangleq s$ is added to the store $S$.

Note that the inference rules of Figure 1 are non-deterministic (i.e., they depend on the chosen constraint of the set $C T$ ). However, they are confluent up to variable renaming (i.e., the chosen transition is irrelevant for computation of 
terminal configurations). This justifies the well-known fact that the least general generalization of two terms is unique up to variable renaming [21].

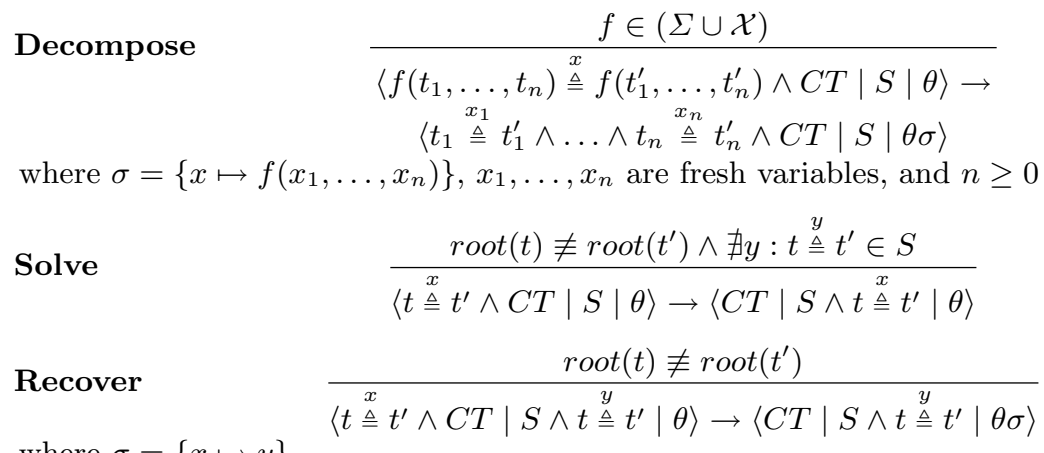

where $\sigma=\{x \mapsto y\}$

Fig. 1. Rules for least general generalization

Example 1. Let $t=f(g(a), g(y), a)$ and $s=f(g(b), g(y), b)$ be two terms. We apply the inference rules of Figure 1 and the substitution obtained by the lgg algorithm is $\theta=\left\{x \mapsto f\left(g\left(x_{4}\right), g(y), x_{4}\right), x_{1} \mapsto g\left(x_{4}\right), x_{2} \mapsto g(y), x_{5} \mapsto y, x_{3} \mapsto\right.$ $\left.x_{4}\right\}$, where the $\operatorname{lgg}$ is $x \theta=f\left(g\left(x_{4}\right), g(y), x_{4}\right)$. Note that variable $x_{4}$ is repeated, to ensure the least general generalization. The execution trace is showed in Figure 2.

Termination and confluence (up to variable renaming) of the transition system $($ Conf,$\rightarrow)$ are straightforward. Soundness and completeness are proved as follows.

Theorem 1. [3] Given terms $t$ and $t^{\prime}$ and a fresh variable $x, u$ is the lgg of $t$ and $t^{\prime}$ iff $\left\langle t \triangleq t^{\prime}|\emptyset| i d\right\rangle \rightarrow^{*}\langle\emptyset|S| \theta\rangle$ and there is a renaming $\rho$ s.t. $u \rho=x \theta$.

Let us mention that the equational generalization algorithm of [3] recalled above can also be used to compute (thanks to associativity and commutativity of lgg) the lgg of an arbitrary set of terms by successively computing the lgg of two elements of the set in the obvious way.

\section{Least General Generalizations modulo E}

When we have an equational theory $E$, the notion of least general generalization has to be broadened, because, there may exist $E$-generalizable terms that do not have a unique least general generalization. Similarly to the dual case of $E$-unification, we have to talk about a set of least general generalizations.

For a set $M$ of terms, we define the set of most specific generalizations of $M$ modulo $E$ as the set of maximal lower bounds of $M$ under $<_{E}$, i.e., $\lg _{E}(M)=$ $\left\{u \mid \forall m \in M, u \leq_{E} m \wedge \nexists u^{\prime}\left(u<_{E} u^{\prime} \wedge \forall m \in M, u^{\prime} \leq_{E} m\right)\right\}$. 


$$
\begin{aligned}
& \lg g(f(g(a), g(y), a), f(g(b), g(y), b)) \\
& \downarrow \text { Initial Configuration } \\
& \langle f(g(a), g(y), a) \triangleq f(g(b), g(y), b)|\emptyset| i d\rangle
\end{aligned}
$$

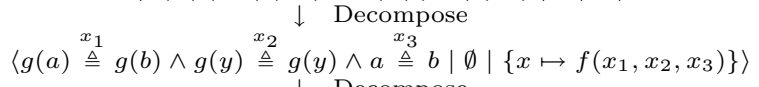

$$
\begin{aligned}
& \downarrow \text { Decompose } \\
& \left\langle a \stackrel{x_{4}}{\triangleq} b \wedge g(y) \stackrel{x_{2}}{\triangleq} g(y) \wedge a \stackrel{x_{3}}{\triangleq} b|\emptyset|\left\{x \mapsto f\left(g\left(x_{4}\right), x_{2}, x_{3}\right), x_{1} \mapsto g\left(x_{4}\right)\right\}\right\rangle \\
& \left\langle g(y) \stackrel{x_{2}}{\triangleq} g(y) \wedge a \stackrel{x_{3}}{\triangleq} b\left|a \stackrel{x_{4}}{\triangleq} b\right|\left\{x \mapsto f\left(g\left(x_{4}\right), x_{2}, x_{3}\right), x_{1} \mapsto g\left(x_{4}\right)\right\}\right\rangle \\
& \downarrow \text { Decompose }
\end{aligned}
$$

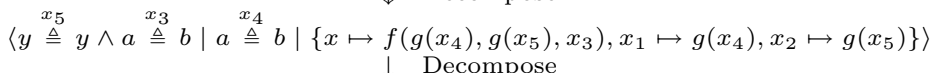

$$
\begin{aligned}
& \left\langle a \stackrel{x_{3}}{\triangleq} b\left|a \stackrel{x_{4}}{\triangleq} b\right|\left\{x \mapsto f\left(g\left(x_{4}\right), g(y), x_{3}\right), x_{1} \mapsto g\left(x_{4}\right), x_{2} \mapsto g(y), x_{5} \mapsto y\right\}\right\rangle \\
& \left\langle\emptyset\left|a \stackrel{x_{4}}{\triangleq} b\right|\left\{x \mapsto f\left(g\left(x_{4}\right), g(y), x_{4}\right), x_{1} \mapsto g\left(x_{4}\right), x_{2} \mapsto g(y), x_{5} \mapsto y, x_{3} \mapsto x_{4}\right\}\right\rangle \\
& \begin{array}{l}
\left.\left.\left.g(y), x_{3}\right), x_{1} \mapsto g\left(x_{4}\right), x_{2} \mapsto g(y), x_{5} \mapsto y\right\}\right\rangle \\
\downarrow \text { Recover } \\
\left.\left.4), x_{1} \mapsto g\left(x_{4}\right), x_{2} \mapsto g(y), x_{5} \mapsto y, x_{3} \mapsto x_{4}\right\}\right\rangle
\end{array}
\end{aligned}
$$

Fig. 2. Computation trace for (syntactic) generalization of terms $f(g(a), g(y), a)$ and $f(g(b), g(y), b)$

Example 2. Consider terms $t=f(a, a, b)$ and $s=f(b, b, a)$ where $f$ is associative and commutative, and $a$ and $b$ are constants. Terms $u=f(x, x, y)$ and $u^{\prime}=$ $f(x, a, b)$ are generalizations of $t$ and $s$ but they are not comparable, i.e., no one is an instance of the other modulo the $A C$ axioms of $f$.

Given a finite set of equations $E$, and given two terms $t$ and $s$, we can always recursively enumerate the set which is by construction a complete set of generalizations of $t$ and $s$. For this, we only need to recursively enumerate all pairs of terms $\left(u, u^{\prime}\right)$ with $t={ }_{E} u$ and $s={ }_{E} u^{\prime}$ and compute $\lg g\left(u, u^{\prime}\right)$. Of course, this set $g e n_{E}(t, s)$ may easily be infinite. However, if the theory $E$ has the additional property that each $E$-equivalence class is finite and can be effectively generated, then the above process becomes a terminating algorithm, generating a finite complete set of generalizations of $t$ and $s$.

In any case, for any finite set of equations $E$, we can always mathematically characterize a minimal complete set of E-generalizations, namely as a set $\operatorname{lgg}_{E}(t, s)$ defined as follows.

Definition 2. Let $t$ and $s$ be terms and let $E$ be an equational theory. A complete set of generalizations of $t$ and $s$ modulo $E$, denoted by $\operatorname{gen}_{E}(t, s)$, is defined as follows: $\operatorname{gen}_{E}(t, s)=\left\{v \mid \exists u, u^{\prime}\right.$ s.t. $\left.t={ }_{E} u \wedge s={ }_{E} u^{\prime} \wedge \operatorname{lgg}\left(u, u^{\prime}\right)=v\right\}$.

The set of least general generalizations of $t$ and $s$ modulo $E$ is defined as follows:

$$
\operatorname{lgg}_{E}(t, s)=\operatorname{maximal}_{<_{E}}\left(\operatorname{gen}_{E}(t, s)\right)
$$

where maximal $_{<_{E}}(S)=\left\{s \in S \mid \nexists s^{\prime} \in S: s<_{E} s^{\prime}\right\}$. Lggs are equivalent modulo renaming and, therefore, we remove from $\operatorname{lgg}_{E}\left(t, t^{\prime}\right)$ renamed versions of terms.

The following result is immediate.

Theorem 2. Given terms $t$ and $s$ in an equational theory $E, \operatorname{lgg}_{E}(t, s)$ is a minimal, correct, and complete set of lggs modulo $E$ of $t$ and $s$ (up to renaming). 
However, note that in general the relation $t<_{E} t^{\prime}$ is undecidable, so that the above set, although definable at the mathematical level, cannot be effectively computed. Nevertheless, when: (i) each $E$-equivalence class is finite and can be effectively generated; and (ii) there is an $E$-matching algorithm, then we also have an effective algorithm for computing $\operatorname{lgg}_{E}(t, s)$, since the relation $t \leq_{E} t^{\prime}$ is precisely the $E$-matching relation.

In summary, when $E$ is finite and satisfies conditions (i) and (ii), the above definitions give us an effective, although horribly inefficient, procedure to compute a finite, minimal, and complete set of least general generalizations $\lg _{E}(t, s)$. This naive algorithm could be used when $E$ consists of associativity and/or commutativity axioms for some functions symbols, because such theories (a special case of our proposed parametric family of theories) all satisfy conditions (i)-(ii). However, when we add identiy axioms, $E$-equivalence classes become infinite, so that the above approach no longer gives us a lgg algorithm modulo $E$.

In the following sections, we do provide a modular, minimal, terminating, sound, and complete algorithm for equational theories containing different axioms such as associativity, commutativity, and identity (and their combinations). The set $l g g_{E}(t, s)$ of least general $E$-generalizations is computed in two phases: (i) first a complete set of $E$-generalizations is computed by the inference rules given below, and then (ii) they are filtered to obtain $l g g_{E}(t, s)$ by using the fact that for all theories $E$ in the parametric family of theories we consider in this paper, there is a matching algorithm modulo $E$. We consider that a given function symbol $f$ in the signature $\Sigma$ obeys a subset of axioms ax $(f) \subseteq\left\{A_{f}, C_{f}, U_{f}\right\}$. In particular, $f$ may not satisfy any such axioms, $a x(f)=\emptyset$.

Let us provide our inference rules for equational generalization in a stepwise manner. First, $a x(f)=\emptyset$, then, $a x(f)=\left\{C_{f}\right\}$, then, $a x(f)=\left\{A_{f}\right\}$, then, $a x(f)=\left\{A_{f}, C_{f}\right\}$, and finally, $\left\{U_{f}\right\} \in a x(f)$. Technically, variables of the original terms are handled in our rules as constants, thus without any attribute, i.e., for any variable $x \in X$, we consider $a x(x)=\emptyset$.

\subsection{Basic rules for least general $E$-generalization}

Let us start with a set of basic rules that are the equational version of the syntactic generalization rules of Section 3. The rule Decompose $E$ applies to function symbols obeying no axioms, $a x(f)=\emptyset$. Specific rules for decomposing constraints involving terms that are rooted by symbols obeying equational axioms, such as ACU and their combinations, are given below.

Concerning the rules Solve $_{E}$ and Recover $_{E}$, the main difference w.r.t. the corresponding syntactic generalization rules given in Section 3 is in the fact that the checks to the store consider the constraints modulo $E$ : in the rules below, we write $t \triangleq{ }^{y} \triangleq t^{\prime} \in \in^{E} S$ to express that there exists $s \triangleq{ }^{\prime} s^{\prime} \in S$ such that $t={ }_{E} s$ and $t^{\prime}={ }_{E} s^{\prime}$.

Finally, regarding the rule Solve $_{E}$, note that this rule cannot be applied to any constraint $t \triangleq s$ such that either $t$ or $s$ are rooted by a function symbol $f$ with $U_{f} \in a x(f)$. For function symbols with an identity element, a speciallytailored rule Expand $_{U}$ is given in Section 4.5 that gives us the opportunity to 


$$
\begin{aligned}
& \operatorname{Decompose}_{E} \frac{f \in(\Sigma \cup \mathcal{X}) \wedge a x(f)=\emptyset}{\left\langle f\left(t_{1}, \ldots, t_{n}\right) \triangleq f\left(t_{1}^{\prime}, \ldots, t_{n}^{\prime}\right) \wedge C T|S| \theta\right\rangle \Rightarrow} \\
& \left\langle t_{1} \triangleq t_{1}^{x_{1}} \wedge \ldots \wedge t_{n} \triangleq t_{n}^{\prime} \wedge C T|S| \theta \sigma\right\rangle \\
& \text { where } \sigma=\left\{x \mapsto f\left(x_{1}, \ldots, x_{n}\right)\right\}, x_{1}, \ldots, x_{n} \text { are fresh variables, and } n \geq 0 \\
& \text { Solve }_{E} \frac{f=\operatorname{root}(t) \wedge g=\operatorname{root}\left(t^{\prime}\right) \wedge f \not \equiv g \wedge U_{f} \notin a x(f) \wedge U_{g} \notin a x(g) \wedge \nexists y: t \stackrel{y}{x} t^{\prime} \in \in^{E} S}{\left\langle t \triangleq t^{\prime} \wedge C T|S| \theta\right\rangle \Rightarrow\left\langle C T\left|\underset{y}{S} \wedge t \triangleq t^{\prime}\right| \theta\right\rangle} \\
& \text { Recover }_{E} \quad \frac{\operatorname{root}(t) \not \equiv \operatorname{root}\left(t^{\prime}\right) \wedge \exists y: t \triangleq t^{\prime} \in^{E} S}{\left\langle t \triangleq t^{\prime} \wedge C T|S| \theta\right\rangle \Rightarrow\langle C T|S| \theta \sigma\rangle} \\
& \text { where } \sigma=\{x \mapsto y\}
\end{aligned}
$$

Fig. 3. Basic rules for least general $E$-generalization

\section{Decompose $_{C}$}

$$
\frac{C_{f} \in a x(f) \wedge A_{f} \notin a x(f) \wedge i \in\{1,2\}}{\left\langle f\left(t_{1}, t_{2}\right) \stackrel{x}{=} f\left(s_{1}, s_{2}\right) \wedge C T|S| \theta\right\rangle \Rightarrow\left\langle t_{1} \stackrel{x_{1}}{=} s_{i} \wedge t_{2} \stackrel{x_{2}}{=} s_{(i \text { mod } 2)+1} \wedge C T|S| \theta \sigma\right\rangle}
$$

where $\sigma=\left\{x \mapsto f\left(x_{1}, x_{2}\right)\right\}$, and $x_{1}, x_{2}$ are fresh variables

Fig. 4. Decomposition rule for a commutative function symbol $f$

solve a constraint (conflict pair) $f\left(t_{1}, t_{2}\right) \triangleq \stackrel{x}{\triangleq} s$, such that $\operatorname{root}(s) \not \equiv f$, with a generalization $f(y, z)$ more specific than $x$, by first introducing the constraint $f\left(t_{1}, t_{2}\right) \stackrel{x}{\triangleq} f(s, e)$.

\subsection{Least general generalization modulo $C$}

In this section we extend the basic set of equational generalization rules by adding a specific inference rule Decompose $_{C}$, given in Figure 4, for dealing with commutativity function symbols. This inference rule replaces the syntactic decomposition inference rule for the case of a binary commutative symbol $f$, i.e., the four possible rearrangements of the terms $f\left(t_{1}, t_{2}\right)$ and $f\left(s_{1}, s_{2}\right)$ are considered. Just notice that this rule is (don't know) non-deterministic, hence all four combinations must be explored.

Example 3. Let $t=f(a, b)$ and $s=f(b, a)$ be two terms where $f$ is commutative, i.e., $C_{f} \in a x(f)$. By applying the rules Solve $_{E}$, Recover $_{E}$, and Decompose $_{C}$ above, we end in a terminal configuration $\langle\emptyset|S| \theta\rangle$, where $\theta=\left\{x \mapsto f(b, a), x_{3} \mapsto\right.$ $\left.b, x_{4} \mapsto a\right\}$, thus we conclude that the $\operatorname{lgg}$ modulo $C$ of $t$ and $s$ is $x \theta=f(b, a)$.

\subsection{Least general generalization modulo $A$}

In this section we provide a specific inference rule Decompose $_{A}$ for handling function symbols obeying the associativity axiom (but not the commutativity one). A specific set of rules for dealing with $\mathrm{AC}$ function symbols is given in the next subsection.

The Decompose $_{A}$ rule is given in Figure 5. We use flattened versions of the terms which use poly-variadic versions of the associative symbols, i.e., being $f$ 


\section{Decompose $_{A}$}

$$
\begin{gathered}
\frac{A_{f} \in a x(f) \wedge C_{f} \notin a x(f) \wedge m \geq 2 \wedge n \geq m \wedge k \in\{1, \ldots,(n-m)+1\}}{\left\langle f\left(t_{1}, \ldots, t_{n}\right) \stackrel{x}{=} f\left(s_{1}, \ldots, s_{m}\right) \wedge C T|S| \theta\right\rangle \Rightarrow} \\
\left\langle f\left(t_{1}, \ldots, t_{k}\right) \stackrel{x_{1}}{=} s_{1} \wedge f\left(t_{k+1}, \ldots, t_{n}\right) \stackrel{x_{2}}{=} f\left(s_{2}, \ldots, s_{m}\right) \wedge C T|S| \theta \sigma\right\rangle
\end{gathered}
$$

where $\sigma=\left\{x \mapsto f\left(x_{1}, x_{2}\right)\right\}$, and $x_{1}, x_{2}$ are fresh variables

Fig. 5. Decomposition rule for an associative (non-commutative) function symbol $f$

an associative symbol,

$$
\operatorname{flat}\left(f\left(t_{1}, \ldots, f\left(s_{1}, \ldots, s_{k}\right), \ldots, t_{n}\right)\right)=\operatorname{flat}\left(f\left(t_{1}, \ldots, s_{1}, \ldots, s_{k}, \ldots, t_{n}\right)\right)
$$

and, otherwise, $\operatorname{flat}\left(f\left(t_{1}, \ldots, t_{n}\right)\right)=f\left(\operatorname{flat}\left(t_{1}\right), \ldots, f l a t\left(t_{n}\right)\right)$. Given an associative symbol $f$ and a term $f\left(t_{1}, \ldots, t_{n}\right)$ we call alien $f$-terms (or simply alien terms) to those terms among $t_{1}, \ldots, t_{n}$ that are not rooted by $f$. In the following, being $f$ an associative poly-varyadic symbol, $f(t)$ represents the term $t$ itself, since symbol $f$ needs at least two arguments. The inference rule of Figure 5 replaces the syntactic decomposition inference rule for the case of an associative function symbol $f$, where all prefixes of $t_{1}, \ldots, t_{n}$ and $s_{1}, \ldots, s_{m}$ are considered. Just notice that this rule is (don't know) non-deterministic, hence all possibilities must be explored.

Note that this is better than generating all terms in the corresponding equivalence class, as explained in Section 4 , since we will eagerly stop in a constraint $t \stackrel{x}{=} f\left(t_{1}, \ldots, t_{n}\right)$ if $\operatorname{root}(t) \not \equiv f$ without considering all the combinations in the equivalence class of $f\left(t_{1}, \ldots, t_{n}\right)$.

We give the rule Decompose $_{A}$ for the case when, in the generalization problem

$s \triangleq t$, the number of alien terms in $s$ is greater than (or equal to) the number of alien terms in $t$. For the other way round, that is, the number of alien terms in $s$ is less than (or equal to) the number of alien terms in $t$, a similar rule would be needed, that we omit since it is perfectly analogous.

Example 4. Let $f(f(a, c), b)$ and $f(c, b)$ be two terms where $f$ is associative, i.e., $A_{f} \in a x(f)$. By applying the rules $S_{\text {Solve }}$, Recover $_{E}$, and Decompose $_{A}$ above, we end in a terminal configuration $\langle\emptyset|S| \theta\rangle$, where $\theta=\left\{x \mapsto f\left(x_{3}, b\right), x_{4} \mapsto b\right\}$, thus we compute that the lgg modulo $A$ of $t$ and $s$ is $f\left(x_{3}, b\right)$. The computation trace is shown in Figure 6.

\subsection{Least general generalization modulo $A C$}

In this section we provide a specific inference rule Decompose $_{A C}$ for handling function symbols obeying both the associativity and commutativity axioms. Note that we use again flattened versions of the terms, as in the associative case of Section 4.3. Actually, the new decomposition rule for the case AC is similar to the decompose inference rule for associative function symbols, except that all permutations of $f\left(t_{1}, \ldots, t_{n}\right)$ and $f\left(s_{1}, \ldots, s_{m}\right)$ are considered. Just notice 


$$
\begin{aligned}
& \operatorname{lgg}_{E}(f(f(a, c), b), f(c, b)), \text { with } E=\left\{A_{f}\right\} \\
& \downarrow \text { Initial Configuration } \\
& \langle f(a, c, b) \stackrel{x}{=} f(c, b)|\emptyset| \emptyset\rangle \\
& \left\langle a \stackrel{x_{1}}{=} c \wedge f(c, b) \stackrel{x_{2}}{=} b|\emptyset|\left\{x \mapsto f\left(x_{1}, x_{2}\right)\right\}\right\rangle\left\langle f(a, c) \stackrel{x_{3}}{=} c \wedge b \stackrel{x_{4}}{=} b \underset{\text { Solve }}{|\emptyset|}\left\{x \mapsto f\left(x_{3}, x_{4}\right)\right\}\right\rangle \\
& \left\langle f(c, b) \stackrel{x_{2}}{=} b\left|a \stackrel{x_{1}}{=} c\right|\left\{x \mapsto f\left(x_{1}, x_{2}\right)\right\}\right\rangle \quad\left\langle b \stackrel{x_{4}}{=} b\left|f(a, c) \stackrel{x_{3}}{=} c\right|\left\{x \mapsto f\left(x_{3}, x_{4}\right)\right\}\right\rangle \\
& \left\langle\emptyset\left|a \stackrel{x_{1}}{=} c \wedge f(c, b) \stackrel{x_{2}}{=} b\right|\left\{x \mapsto f\left(x_{1}, x_{2}\right)\right\}\right\rangle\left\langle\emptyset\left|f(a, c) \stackrel{x_{3}}{=} c\right|\left\{x \mapsto f\left(x_{3}, b\right), x_{4} \mapsto b\right\}\right\rangle \\
& \begin{array}{l}
\searrow \operatorname{maximal}_{\mathcal{A}_{A}} \swarrow \\
\left\{x \mapsto f\left(x_{3}, b\right), x_{4} \mapsto b\right\}
\end{array}
\end{aligned}
$$

Fig. 6. Computation trace for A-generalization of terms $f(f(a, c), b)$ and $f(c, b))$.

\section{Decompose $_{A C}$}

$$
\begin{aligned}
& \frac{\left\{A_{f}, C_{f}\right\} \subseteq a x(f) \wedge n \geq m \wedge\left\{i_{1}, \ldots, i_{m-1}\right\} \uplus\left\{i_{m}, \ldots, i_{n}\right\}=\{1, \ldots, n\}}{\left\langle f\left(t_{1}, \ldots, t_{n}\right) \stackrel{x}{=} f\left(s_{1}, \ldots, s_{m}\right) \wedge C|S| \theta\right\rangle \Rightarrow} \\
& \left\langle t_{i_{1}} \stackrel{x_{1}}{=} s_{1} \wedge \ldots \wedge t_{i_{m-1}} \stackrel{x_{m-1}}{=} s_{m-1} \wedge f\left(t_{i_{m}}, \ldots, t_{i_{n}}\right) \stackrel{x_{m}}{=} s_{m} \wedge C|S| \theta \sigma\right\rangle
\end{aligned}
$$

where $\sigma=\left\{x \mapsto f\left(x_{1}, \ldots, x_{m}\right)\right\}$, and $x_{1}, \ldots, x_{m}$ are fresh variables

Fig. 7. Decomposition rule for an associative-commutative function symbol $f$

that this rule is (don't know) non-deterministic, hence all possibilities must be explored.

Similarly to the rule Decompose $_{A}$, we give the rule Decompose $_{A C}$ for the case when, in the generalization problem $s \stackrel{x}{\triangleq} t$, the number of alien terms in $s$ is greater than or equal to the number of alien terms in $t$. For the other way round, a similar rule would be needed, that we omit since it is perfectly analogous. To simplify, we write $\left\{i_{1}, \ldots, i_{k}\right\} \Psi\left\{i_{k+1}, \ldots, i_{n}\right\}=\{1, \ldots, n\}$ to denote that the sequence $\left\{i_{1}, \ldots, i_{n}\right\}$ is a permutation of the sequence $\{1, \ldots, n\}$ and, given an element $k \in\{1, \ldots, n\}$, we split the sequence $\left\{i_{1}, \ldots, i_{n}\right\}$ in the two parts, $\left\{i_{1}, \ldots, i_{k}\right\}$ and $\left\{i_{k+1}, \ldots, i_{n}\right\}$.

Example 5. Let $t=f(a, f(a, b))$ and $s=f(f(b, b), a)$ be two terms where $f$ is associative and commutative, i.e., $\left\{A_{f}, C_{f}\right\} \subseteq a x(f)$. By applying the rules Solve $_{E}$, Recover $_{E}$, and Decompose $A C$ above, we end in two terminal configurations whose respective substitution components are $\theta_{1}=\left\{x \mapsto f\left(x_{1}, x_{1}, x_{3}\right), x_{2} \mapsto\right.$ $\left.x_{1}\right\}$ and $\theta_{2}=\left\{x \mapsto f\left(x_{4}, a, b\right), x_{5} \mapsto a, x_{6} \mapsto b\right\}$, thus we compute that the lggs modulo $A C$ of $t$ and $s$ are $f\left(x_{1}, x_{1}, x_{3}\right)$ and $f\left(x_{4}, a, b\right)$. The corresponding computation trace is shown in Figure 8.

\subsection{Least general generalization modulo $U$}

Finally, let us introduce the inference rule of Figure 9 for handling function symbols $f$ which have an identity element $e$. This rule considers the identity axioms in a rather lazy or on-demand manner. The rule corresponds to the case when the root symbol $f$ of the term $t$ in the left-hand side of the constraint $t \stackrel{x}{=} s$ obeys the unity axioms. A companion rule for handling the case when the root 


$$
\begin{aligned}
& \operatorname{lgg}_{E}(f(a, f(a, b)), f(f(b, b), a)), \text { with } E=\left\{C_{f}, A_{f}\right\} \\
& \downarrow \text { Initial Configuration } \\
& \langle f(a, a, b) \stackrel{x}{=} f(b, b, a)|\emptyset| i d\rangle \\
& \left\langle a \stackrel{x_{1}}{=} b \wedge a \stackrel{x_{2}}{=} b \wedge b \stackrel{x_{3}}{=} a|\emptyset|\left\{x \mapsto f\left(x_{1}, x_{2}, x_{3}\right)\right\}\right\rangle \quad\left\langle a \stackrel{x_{4}}{=} b \wedge a \stackrel{x_{5}}{=} a \wedge b \stackrel{x_{6}}{=} b|\emptyset|\left\{x \mapsto f\left(x_{4}, x_{5}, x_{6}\right)\right\}\right\rangle \\
& \left\langle a \stackrel{x_{2}}{=} b \wedge b \stackrel{x_{3}}{=} a\left|\underset{\downarrow}{\stackrel{x_{1}}{=}} b\right|\left\{x \mapsto f\left(x_{1}, x_{2}, x_{3}\right)\right\}\right\rangle \quad\left\langle a \stackrel{x_{5}}{=} a \wedge b \stackrel{x_{6}}{=} b\left|\underset{\downarrow}{\mid a x_{4}}=\underset{\text { Decompore }}{=} b\right|\left\{x \mapsto f\left(x_{4}, x_{5}, x_{6}\right)\right\}\right\rangle \\
& \left.\left.\left\langle b \stackrel{x_{3}}{=} a\left|a \stackrel{x_{1}}{=} b\right|\left\{x \mapsto \underset{\text { Solve }}{f}\left(x_{1}, x_{1}, x_{3}\right), x_{2} \mapsto x_{1}\right\}\right\rangle \quad\left\langle b \stackrel{x_{6}}{=} b\right| a \stackrel{x_{4}}{=} b \underset{\downarrow}{\mid} \underset{\text { Decompose }}{\{x} f\left(x_{4}, a, x_{6}\right), x_{5} \mapsto a\right\}\right\rangle \\
& \left\langle\emptyset\left|a \stackrel{x_{1}}{=} b \wedge b \stackrel{x_{3}}{=} a\right|\left\{x \mapsto f\left(x_{1}, x_{1}, x_{3}\right), x_{2} \mapsto x_{1}\right\}\right\rangle\left\langle\emptyset\left|a \stackrel{x_{4}}{=} b\right|\left\{x \mapsto f\left(x_{4}, a, b\right), x_{5} \mapsto a, x_{6} \mapsto b\right\}\right\rangle \\
& \left\{x \mapsto f\left(x_{1}, x_{1}, x_{3}\right), x_{2} \mapsto x_{1}\right\} \text { and }\left\{x \mapsto f\left(x_{4}, a, b\right), x_{5} \mapsto a, x_{6} \mapsto b\right\}
\end{aligned}
$$

Fig. 8. Computation trace for AC-generalizations of terms $f(a, f(a, b))$ and $f(f(b, b), a)$.

$$
\operatorname{Expand}_{U} \frac{U_{f} \in a x(f) \wedge \operatorname{root}(t) \equiv f \wedge \operatorname{root}(s) \not \equiv f \wedge s^{\prime} \in\{f(e, s), f(s, e)\}}{\langle t \stackrel{x}{=} s \wedge C T|S| \theta\rangle \Rightarrow\left\langle t \stackrel{x}{=} s^{\prime} \wedge C T|S| \theta\right\rangle}
$$

Fig. 9. Inference rule for expanding function symbol $f$ with identity element $e$

symbol of the term $s$ in the right-hand side obeys the unity axiom is omitted, that is perfectly analogous.

Example 6. Let $t=f(a, b, c, d)$ and $s=f(a, c)$ be two terms where $\left\{A_{f}, C_{f}, U_{f}\right\} \subseteq$ ax $(f)$. By applying the rules Solve $_{E}$, Recover $_{E}$, Decompose $_{A C}$, and Expand $_{U}$ above, we end in a terminal configuration $\langle\emptyset|S| \theta\rangle$, where $\theta=\left\{x \mapsto f\left(a, f\left(c, f\left(x_{5}, x_{6}\right)\right)\right), x_{1} \mapsto a, x_{2} \mapsto f\left(c, f\left(x_{5}, x_{6}\right)\right), x_{3} \mapsto c, x_{4} \mapsto\right.$ $\left.f\left(x_{5}, x_{6}\right)\right\}$, thus we compute that the $\operatorname{lgg}$ modulo $A C U$ of $t$ and $s$ is $f\left(a, c, x_{5}, x_{6}\right)$. The computation trace is shown in Figure 10.

\subsection{A general ACU-generalization method}

For the general case when different function symbols satisfying different associativity and/or commutativity and/or identity axioms are considered, we can use the rules above all together, with no need whatsoever for any changes or adaptations.

The key property of all the above inference rules is their locality: they are local to the given top function symbol in the left term (or right term in some cases) of the constraint they are acting upon, irrespective of what other function symbols and what other axioms may be present in the given signature $\Sigma$ and theory $E$. Such a locality means that these rules are modular, in the sense that they do not need to be changed or modified when new function symbols are added to the signature and new $A$, and/or $C$, and/or $U$ axioms are added to $E$. However, when new axioms are added to $E$, some rules that applied before (for example decomposition for an $f$ which before satisfied $a x(f)=\emptyset$, but now has $a x(f) \neq \emptyset$ ) may not apply, and, conversely, some rules that did not apply before now may apply (because new axioms are added to $f$ ). But the rules themselves do not change! They are the same and can be used to compute the set of lggs of two terms modulo any theory $E$ in the parametric family $\mathbb{E}$ of theories of 


$$
\begin{aligned}
& \operatorname{lgg}_{E}(f(a, b, c, d), f(a, c)), \text { with } E=\left\{C_{f}, A_{f}, U_{f}\right\} \\
& \downarrow \text { Initial Configuration } \\
& \langle f(a, b, c, d) \stackrel{x}{=} f(a, c)|\emptyset| i d\rangle \\
& \downarrow \text { Decompose } A C \text { (Other permutations are not shown) } \\
& \left\langle a \stackrel{x_{1}}{=} a \wedge f(b, c, d) \stackrel{x_{2}}{=} c|\emptyset|\left\{x \mapsto f\left(x_{1}, x_{2}\right)\right\}\right\rangle \\
& \downarrow \text { Decompose } \\
& \left\langle f(b, c, d) \stackrel{x_{2}}{=} c|\emptyset|\left\{x \mapsto f\left(a, x_{2}\right), x_{1} \mapsto a\right\}\right\rangle \\
& \downarrow \operatorname{Expand}_{U} \\
& \left\langle f(b, c, d) \stackrel{x_{2}}{=} f(c, e)|\emptyset|\left\{x \mapsto f\left(a, x_{2}\right), x_{1} \mapsto a\right\}\right\rangle \\
& \downarrow \text { Decompose }_{A C} \text { (Other permutations are not shown) } \\
& \left\langle c \stackrel{x_{3}}{=} c \wedge f(b, d) \stackrel{x_{4}}{=} e|\emptyset|\left\{x \mapsto f\left(a, f\left(x_{3}, x_{4}\right)\right), x_{1} \mapsto a, x_{2} \mapsto f\left(x_{3}, x_{4}\right)\right\}\right\rangle \\
& \downarrow \text { Decompose } \\
& \left\langle f(b, d) \stackrel{x_{4}}{=} e|\emptyset|\left\{x \mapsto f\left(a, f\left(c, x_{4}\right)\right), x_{1} \mapsto a, x_{2} \mapsto f\left(c, x_{4}\right), x_{3} \mapsto c\right\}\right\rangle \\
& \downarrow \operatorname{Expand}_{U} \\
& \left\langle f(b, d) \stackrel{x_{4}}{=} f(e, e)|\emptyset|\left\{x \mapsto f\left(a, f\left(c, x_{4}\right)\right), x_{1} \mapsto a, x_{2} \mapsto f\left(c, x_{4}\right), x_{3} \mapsto c\right\}\right\rangle \\
& \downarrow \text { Decompose }_{A C} \text { (Other permutations are not shown) } \\
& \left\langle b \stackrel{x_{5}}{=} e \wedge d \stackrel{x_{6}}{=} e|\emptyset|\left\{x \mapsto f\left(a, f\left(c, f\left(x_{5}, x_{6}\right)\right)\right), x_{1} \mapsto a, x_{2} \mapsto f\left(c, f\left(x_{5}, x_{6}\right)\right), x_{3} \mapsto c, x_{4} \mapsto f\left(x_{5}, x_{6}\right)\right\}\right\rangle \\
& \left\langle d \stackrel{x_{6}}{=} e\left|b \stackrel{x_{5}}{=} e\right|\left\{x \mapsto f\left(a, f\left(c, f\left(x_{5}, x_{6}\right)\right)\right), x_{1} \mapsto a, x_{2} \mapsto f\left(c, f\left(x_{5}, x_{6}\right)\right), x_{3} \mapsto c, x_{4} \mapsto f\left(x_{5}, x_{6}\right)\right\}\right\rangle \\
& \left\langle\emptyset\left|b \stackrel{x_{5}}{=} e \wedge d \stackrel{x_{6}}{=} e\right|\left\{x \mapsto f\left(a, f\left(c, f\left(x_{5}, x_{6}\right)\right)\right), x_{1} \mapsto a, x_{2} \mapsto f\left(c, f\left(x_{5}, x_{6}\right)\right), x_{3} \mapsto c, x_{4} \mapsto f\left(x_{5}, x_{6}\right)\right\}\right\rangle \\
& \left\{x \mapsto f\left(a, f\left(c, f\left(x_{5}, x_{6}\right)\right)\right), x_{1} \mapsto a, x_{2} \mapsto f\left(c, f\left(x_{5}, x_{6}\right)\right), x_{3} \mapsto c, x_{4} \mapsto f\left(x_{5}, x_{6}\right)\right\}
\end{aligned}
$$

Fig. 10. Computation trace for U-generalization of terms $f(a, b, c, d)$ and $f(a, c)$.

the form $E=\bigcup_{f \in \Sigma} a x(f)$, where $a x(f) \subseteq\left\{A_{f}, C_{f}, U_{f}\right\}$. Termination of the algorithm is straightforward.

Theorem 3. Every derivation stemming from an initial configuration $\langle t \triangleq s|$ $\emptyset \mid$ id $\rangle$ terminates.

Let us prove the correctness and completeness of our algorithm.

Theorem 4. Given terms $t$ and $s$, an equational theory $E \in \mathbb{E}$, and a fresh variable $x$, then $\operatorname{lgg}_{E}(t, s)=\operatorname{maximal}_{<_{E}}\left(\left\{x \theta \mid\langle t \stackrel{x}{\triangleq} s|\emptyset| i d\rangle \Rightarrow^{*}\langle\emptyset|S| \theta\rangle\right\}\right)$, up to renaming.

The ACU least general generalization algorithm presented here has been implemented in Maude [11], with a Web interface written in Java. The core of the tool contains about 200 lines of Maude code. The Web tool is publicly available together with a set of examples at the following url: http://www.dsic . upv.es/users/elp/toolsMaude/Lgg2.html.

\section{Conclusions and Future Work}

We have presented a modular equational generalization algorithm that computes a minimal and complete set of least general generalizations for two terms modulo any combination of associativity, commutativity and identity axioms for the binary symbols in the theory. Our algorithm is directly applicable to any untyped declarative language and reasoning systems. However, it would be highly 
desirable to support generalization modulo equational theories $(\Sigma, E)$ where $\Sigma$ is a typed signature such as for example an order-sorted signature, since a number of rule-based languages such as ASF+SDF [8], Elan [9], OBJ [18], CafeOBJ [14], and Maude [11] support order-sorted or many-sorted signatures. All existing generalization algorithms, with the exception of [27] and [1], assume an untyped setting. Moreover, the algorithm for generalization in the calculus of constructions of [27] cannot be used for order-sorted theories. In [3], we have developed an order-sorted generalization algorithm for the case where the set $E$ of axioms is empty. It would be very useful to combine the order-sorted and the $E$-generalization inference systems into a single calculus supporting both types and equational axioms. However, this combination seems to us non-trivial and is left for future work.

In our own work, we plan to use the above-mentioned order-sorted equational generalization algorithm as a key component of a narrowing-based partial evaluator $(\mathrm{PE})$ for programs in order-sorted rule-based languages such as OBJ, CafeOBJ, and Maude. This will make available for such languages useful narrowing-driven PE techniques developed for the untyped setting in, e.g., $[4,5]$. We are also considering adding this generalization mechanism to an inductive theorem prover such a Maude's ITP [12] to support automatic conjecture of lem-

mas. This will provide a typed analogue of similar automatic lemma conjecture mechanisms in untyped inductive theorem provers such as Nqthm [10] and its ACL2 successor [20].

\section{References}

1. H. Aït-Kaci and Y. Sasaki. An Axiomatic Approach to Feature Term Generalization. In Proc. 12th European Conf. on Machine Learning EMCL '01, pages 1-12, London, UK, 2001. Springer-Verlag.

2. M. Alpuente, S. Escobar, J. Meseguer, and P. Ojeda. A modular Equational Generalization Algorithm. Technical Report DSIC-II/6/08, DSIC-UPV, 2008.

3. M. Alpuente, S. Escobar, J. Meseguer, and P. Ojeda. Order-Sorted Generalization. Electr. Notes Theor. Comput. Sci., 2008. to appear.

4. M. Alpuente, M. Falaschi, and G. Vidal. Partial evaluation of functional logic programs. ACM Trans. Program. Lang. Syst., 20(4):768-844, 1998.

5. M. Alpuente, S. Lucas, M. Hanus, and G. Vidal. Specialization of functional logic programs based on needed narrowing. TPLP, 5(3):273-303, 2005.

6. F. Baader and W. Snyder. Unification theory. In Handbook of Automated Reasoning. Elsevier, 1999.

7. F. Belli and O. Jack. Declarative paradigm of test coverage. Softw. Test., Verif. Reliab., 8(1):15-47, 1998.

8. J.A. Bergstra, J. Heering, and P. Klint. Algebraic Specification. ACM Press, 1989.

9. P. Borovanský, C. Kirchner, H. Kirchner, and P.-E. Moreau. ELAN from a rewriting logic point of view. Theoretical Computer Science, 285:155-185, 2002.

10. R. Boyer and J. Moore. A Computational Logic. Academic Press, 1980.

11. M. Clavel, F. Durán, S. Eker, P. Lincoln, N. Martí-Oliet, J. Meseguer, and C. Talcott. All About Maude - A High-Performance Logical Framework. Springer-Verlag New York, Inc., Secaucus, NJ, USA, 2007. 
12. M. Clavel and M. Palomino. The ITP tool's manual. Universidad Complutense, Madrid, April 2005, http://maude.sip.ucm.es/itp/.

13. V. Cortier, S. Delaune, and P. Lafourcade. A Survey of Algebraic Properties used in Cryptographic Protocols. Journal of Computer Security, 14(1):1-43, 2006.

14. R. Diaconescu and K. Futatsugi. CafeOBJ Report, volume 6 of AMAST Series in Computing. World Scientific, AMAST Series, 1998.

15. S. Escobar, J. Meseguer, and P. Thati. Narrowing and rewriting logic: from foundations to applications. Electr. Notes Theor. Comput. Sci., 177:5-33, 2007.

16. S. Escobar, C. Meadows, and J. Meseguer. A rewriting-based inference system for the NRL Protocol Analyzer and its meta-logical properties. Theoretical Computer Science, 367(1-2):162-202, 2006.

17. J. P. Gallagher. Tutorial on specialisation of logic programs. In Proc. PEPM '93, pages 88-98, ACM, 1993.

18. J. Goguen, T. Winkler, J. Meseguer, K. Futatsugi, and J.-P. Jouannaud. Introducing OBJ. In Software Engineering with OBJ: Algebraic Specification in Action, pages 3-167. Kluwer, 2000.

19. G. Huet. Resolution d'Equations dans des Langages d'Order 1, 2, ., $\omega . \mathrm{PhD}$ thesis, Univ. Paris VII, 1976.

20. M. Kaufmann, P. Manolios, and J.S Moore. Computer-Aided Reasoning: An Approach. Kluwer, 2000.

21. J.-L. Lassez, M. J. Maher, and K. Marriott. Unification Revisited. In J. Minker, editor, Foundations of Deductive Databases and Logic Programming, pages 587625. Morgan Kaufmann, Los Altos, Ca., 1988.

22. J. Meseguer. Conditioned rewriting logic as a united model of concurrency. Theor. Comput. Sci., 96(1):73-155, 1992.

23. J. Meseguer. Membership algebra as a logical framework for equational specification. In Proc. WADT'97, pages 18-61, 1997.

24. T. Æ. Mogensen. Glossary for partial evaluation and related topics. Higher-Order and Symbolic Computation, 13(4), 2000.

25. S. Muggleton. Inductive Logic Programming: Issues, Results and the Challenge of Learning Language in Logic. Artif. Intell., 114(1-2):283-296, 1999.

26. B. Østvold. A functional reconstruction of anti-unification. Technical Report DART/04/04, Norwegian Computing Center, 2004. Available at http: //publications.nr.no/nr-notat-dart-04-04.pdf.

27. F. Pfenning. Unification and anti-unification in the calculus of constructions. In Proc. LICS'91, pages 74-85. IEEE Computer Society, 1991.

28. G.D. Plotkin. A note on inductive generalization. In Machine Intelligence, volume 5, pages 153-163. Edinburgh University Press, 1970.

29. G.D. Plotkin. A structural approach to operational semantics. J. Log. Algebr. Program., 60-61:17-139, 2004.

30. R.J. Popplestone. An experiment in automatic induction. In Machine Intelligence, volume 5, pages 203-215. Edinburgh University Press, 1969.

31. J. Reynolds. Transformational systems and the algebraic structure of atomic formulas. Machine Intelligence, 5:135-151, 1970.

32. J.H. Siekmann. Unification Theory. Journal of Symbolic Computation, 7:207-274, 1989.

33. TeReSe, editor. Term Rewriting Systems. Cambridge University Press, Cambridge, 2003. 